\title{
NUMERICAL SIMULATIONS OF JETS FROM ACCRETION DISKS
}

\author{
RALPH E. PUDRITZ \\ Canadian Institute for Theoretical Astrophysics, University of \\ Toronto, Toronto, Ontario, Canada M5S $3 \mathrm{H} 8$ \\ AND \\ RACHID OUYED \\ Department of Physics and Astronomy, McMaster University \\ Hamilton, Ontario, Canada L8S 4M1
}

\begin{abstract}
Hydromagnetic disk winds have great potential as a general theory for the production and collimation of astrophysical jets in both protostellar and black hole environments. We first review the analytic stationary theory of these outflows as well as recent numerical simulations of MHD disk winds. We then focus on simulations that we have done on winds from magnetized disks using the ZEUS 2-D code of Stone and Norman. We treat the Keplerian disk as a fixed platform throughout the simulations. We show that both stationary and episodic, jet-like outflows are driven from disks depending upon their magnetic structure and mass loss rates.
\end{abstract}

\section{Introduction}

The nature and origin of bipolar outflows and jets in protostellar systems continues to be an important and lively area of research seventeen years after the seminal discovery paper of Snell et al. (1980). Many significant observational advances over this period of time leave little doubt that outflows are associated with the process of star formation itself. This connection between star formation and outflow is well seen in the recently discovered, so-called Class 0 sources which are probably the best candidates yet found for protostars (André et al. 1993). Class 0 sources are associated with highly collimated outflows whose mechanical luminosities are comparable to the source bolometric luminosity, $L_{\text {mech }} \simeq L_{b o l}$. This suggests that the efficient 
conversion of gravitational binding energy, released during accretion, into the mechanical energy of the jet is occurring at this early stage in star formation. Moreover, outflows continue to play an important role during the entire collapse and accretion disk formation stage; the evidence shows that outflows persist for at least $10^{5}$ years (Parker, Padman, and Scott, 1991). The role of outflows has yet to be fully integrated into our theories of star formation (see review; Pudritz, McLaughlin, and Ouyed 1997).

The driver for molecular outflows on larger scales is believed to be something akin to optical jets (Masson and Chernin, 1992). The HST images of the optical jet in HH30 (Stapelfeldt 1996) reveal that these jets are already well collimated on scales of $15 \mathrm{AU}$. This jet contains a series of knots whose proper motions have been measured. In fact the observations of a large sample of optical jets (eg. Reipurth 1989, Eislöffel and Mundt 1994) indicates that nature rarely, if ever, produces the stationary outflows which are the domain of much intensive theoretical work (eg. Blandford and Payne, 1982; Pudritz and Norman, 1986, Königl 1989, Heyvaerts and Norman 1989, Lovelace et al., Li 1995, to cite only few). Jets observed in protostars and AGNs are highly time variable and episodic. As to the question of how astrophysical jets are driven, there is now substantial evidence that they are associated with accretion disks (eg. Cabrit and André 1991; Calvet, Hartmann, and Kenyon 1993, Edwards, Ray, and Mundt 1993) which are known to exist around most, if not all young stellar objects (eg. Beckwith et al. 1990). This is seen in the HST image of the jet in HH30 as an example, where a disk is clearly present in the system. In general, it appears that whenever a jet or outflow is detected in protostellar environments, there is good evidence that an accretion disk is also present. This correlation has recently been extended to the case of Class 0 sources with the detection of a disk in VLA 1623 (Pudritz et al. 1996).

Current models for the central engines that produce and collimate jets emphasize the importance of magnetized rotors. Such a mechanism can accelerate gas in systems whose radiation fields are known observationally to be insufficient to provide the observed thrust associated with outflows. Two good candidates for such rotors are (i) magnetized Keplerian disks, or, (ii) the interaction region between a stellar magnetosphere and a surrounding disk (the so-called X-celerator). A complete theory of outflows in protostellar systems must necessarily address both of these aspects of the problem. The most important difference between these two types of models lies in the role played by the central object. For disk winds, (eg. reviews by Königl and Ruden 1993, Pudritz, Pelletier, and Gomez de Castro 1991) only the mass of the central object is important; it dictates the depth of the gravitational potential well and hence the overall energetics of the outflow. In the second class of models on the other hand (eg. Shu et al. 1994), the 
outflow also depends upon the presence of a sufficiently strong magnetic field on the central star. Given that stars of all types are now known to be associated with bipolar outflows, this implies that a wide class of stellar types generate strong magnetic fields in their pre-main-sequence stage. Since energetic, highly collimated outflows are also associated with black holes, both massive as in AGNs (eg. Blandford 1990) as well as stellar mass holes (eg. Hjellming and Rupen, 1995), a universal theory for jet production is in principle possible in the context of disk wind models.

In this contribution, we review some of the recent results of simulations of magnetized winds from accretion disks. We then describe our own simulations of axisymmetric disk wind simulations in some detail. The general reader may consult Ouyed, Pudritz, and Stone (1997, hereafter OPS) for the basic results. We have found that both stationary (details in Ouyed and Pudritz 1997a, OPI) and episodic outflows (details in Ouyed and Pudritz $1997 \mathrm{~b}$, OPII) can be produced depending on the magnetic configuration threading the disk and on the rate at which mass enters the disk corona from the disk (details in Ouyed and Pudritz 1997c, OPIII).

\section{Current Picture of Disk Winds}

Theoretical models of hydromagnetic disk winds originated with the paper of Blandford and Payne (1982, hereafter BP) who analyzed the structure of stationary, self-similar outflows. Their theory extended the models of winds from magnetized rotating stars to the case of accretion disks. One of the simplest predictions of this model arises from Bernoulli's theorem; if magnetic field lines threading a disk make an angle $\theta_{o} \leq 60^{\circ}$ with respect to the surface of the disk, then no outflow should occur. If this condition is satisfied, then gas is accelerated along the field line; in effect one has a rather elegant sort of centrifuge. Gas will continue to be accelerated as long as it can be held in co-rotation with the disk. Thus, the role of the magnetic field is interesting; it is the deformable tool that extracts the gravitational potential energy of particles in a disk allowing those particles to spiral slowly inwards. No magnetic energy is actually used in this process. The Poynting flux associated with the outflow can be traced to the gravitational potential energy that is tapped by the torque exerted upon the disk by the threading magnetic field. The torque extracts angular momentum of material in the disk enabling the accretion process to occur. It is easy to show using the disk angular momentum equation that the torque exerted by the field and the wind necessarily makes the disk accretion rate proportional to the wind mass loss rate from the disk.

Consider a field line threading the disk at some radius $r_{o}$. Acceleration of the gas on any field line occurs predominantly out to the Alfvén point, at 
the radius $r_{A}$ on that field line. The collection of all of these points for field lines threading the disk defines the Alfvén surface $r_{A}\left(r_{o}\right)$ of the wind. On this surface, the kinetic energy density in the poloidal motion of the outflow just equals the energy density in the poloidal component of the field, ie., the outflow speed equals the Alfvén speed. The importance of the Alfvén surface can be seen in another guise; it is directly related to the accretion rate through the disk. The disk angular momentum equation yields the relation $\dot{M}_{a}=<\left(r_{A}\left(r_{o}\right) / r_{o}\right)^{2}>\dot{M}_{w}$, where the appropriate average of the lever arm occurs as one sums up outflow over the entire disk surface. Beyond the Alfvén surface, the field is not able to maintain the rigid co-rotation of the gas with the disk. Nonetheless, some centrifugal acceleration continues out to the point where the outflow speed reaches the fast magnetosonic speed, generally the highest signal speed that occurs in magnetized gas. The surface consisting of all these critical points in the outflow is the fast magnetosonic ( $\equiv \mathrm{FM}$ ) surface. Beyond the FM surface, the flow can no longer communicate with the disk. The dynamics of the jet in this far field regime appears to be dominated by the toroidal magnetic field.

The magnetic structure of the outflow gradually changes as the Alfvén point is passed. Because of the inertia of matter, gas on field lines beyond $r_{A}$ falls behind the rotation rate of the disk as mentioned, and a toroidal field is produced. This field dominates the dynamics of the flow beyond the FM surface. It provides the intrinsic collimation mechanism of outflows through the associated $\mathbf{J}_{\mathbf{p}} \times \mathbf{B}_{\phi}$ Lorentz force which squeezes the flow radially inwards towards the outflow axis.

The discussion so far has assumed that the magnetic field is frozen into the disk plasma. In steady state models, this assumption cannot be strictly valid however. Matter in the disk must cross field lines as it moves radially inwards. If this did not happen in such models, the magnetic flux would be dragged into small disk radii, accumulate there, and eventually shut off the accretion flow itself. Thus, steady state theory involves a balancing act between the outward diffusion of the magnetic field, and the inward drag via the accretion flow (eg. Lubow, Papaloizou, and Pringle 1994). Some dissipation must occur in the magnetized accretion process. Indeed, these dissipative processes are important in determining exactly how the gas in the disk is divided between the small amount that is ejected by the wind, and the far larger portion that accretes onto the star (eg. Wardle and Königl 1993, Ferreira and Pelletier 1995).

One of the theoretical issues left unresolved by the theory is how the disk's magnetic field is distributed (ie. as a function of disk radius). This is a boundary condition for the outflow problem and in all generality, the physics of the accretion process must specify this function in some way. If one invokes some additional mathematical assumption, such as self- 
similarity, then this flux distribution function is fixed (eg BP). There is no physical reason why self-similarity should occur however. The production of an ordered, large scale field could proceed by dynamo action (eg Pudritz 1981, Tout and Pringle 1992, Stone et al. 1996), global modes in the Balbus-Hawley instability (Curry, Pudritz, and Sutherland 1994), or by dragging in a field from the external medium (eg. Lubow et al. 1994, Reys-Ruis and Stepinski 1996). A class of tractable non-self-similar models was found by Pelletier and Pudritz (1992) for outflows in a large variety of magnetic flux distributions.

\subsection{NUMERICAL SIMULATIONS}

These analytical models, while useful in revealing the physics of outflow, cannot address the observations in any direct way. The reason is that real astrophysical jets are episodic, not stationary. This strongly suggests that one needs to use time-dependent numerical simulations in order to investigate this physics. One would like to both confirm the steady state models by using numerical simulations and to explore the presumably much larger class of models that are truly time-dependent.

Not many published MHD disk wind calculations are currently available. The existing calculations divide into two general types; (i) in which the dynamics of the disk is also followed (Shibata and Uchida 1986, Stone and Norman 1994, Bell and Lucek 1995), and (ii) in which evolution of the accretion disk is ignored. In the latter class, the disk acts like a platform providing fixed boundary conditions for the problem (eg. Ustyugova et al. 1995, OPS). In axisymmetric simulations, the Balbus and Hawley (1991) instability leads to very rapid contraction of the disk for models of type (i). This leads to the formation of a transient mass ejection which follows from the winding up of the field lines threading the radially collapsing disk (eg. Uchida and Shibata 1985). Thus, it is not clear from such simulations that outflows would continue over the $10^{5}$ years of outflow in protostellar systems. Models of type (ii), while limited by the neglect of disk evolution, have the virtue that one can isolate outflow physics from disk dynamics. Presumably real disks retain their Keplerian character, which makes this assumption palatable.

\section{Description of our Model}

Our simulations are solutions to the time-dependent MHD equations in cylindrical coordinates $(r, \phi, z)$ calculated with the ZEUS-2D code of Stone and Norman $(1992 \mathrm{a}, \mathrm{b})$. The model consists of a point mass representing a star or black hole, the surface of a surrounding accretion disk which is taken to have an inner radius $r_{i}$ (this can be either the surface of the star, the 
magnetopause radius of a star with the disk, or the last stable Kepler-like orbit around a black hole), an initial disk corona which is in hydrostatic equilibrium within the gravitational field of the central object as well as in pressure balance with the disk below, and an initial magnetic field that threads the disk and corona (see OPS). The gas has an assumed polytropic $(\gamma=5 / 3)$ equation of state. The equilibrium density of the corona is then $\rho \propto\left(r^{2}+z^{2}\right)^{-3 / 4}$. This density structure has the same form as that expected for a collapsing singular isothermal sphere (Shu 1977).

We choose two initial magnetic configurations which are current free; $\mathbf{J}=0$. Surfaces of constant magnetic flux are specified by the level surfaces of the scalar function $A_{\phi}$ where $\mathbf{B}=\nabla \times\left(A_{\phi} \mathbf{e}_{\phi}\right)$. We used two types of initial magnetic configurations $A_{\phi}$; (i) a "potential field" solution corresponding to a vacuum field that has a conducting plate as its boundary (eg. Cao and Spruit 1994); and (ii) a uniform vertical field.

We set the initial toroidal field component $B_{\phi}$ and gas velocity everywhere in the corona equal to zero. We avoid the use of a softening parameter for the (Newtonian) potential and take particular care to establish the disk corona in numerical hydrostatic equilibrium so that there are no initial motions.

The Keplerian accretion disk surface (the base of the corona at $z=0$ ) provides fixed boundary conditions for the velocity at every disk radius; the rotational speed is Keplerian $v_{K}$ and since any radial inflow speed is expected to be tiny compared to this, we ignore it. Matter is introduced into the corona (active zones of the grid) from the disk at very small injection speeds $v_{i n j}$.; typically $v_{i n j .} \simeq 10^{-3} v_{K}$ (one hundredth of the disk's sound speed). The disk's toroidal magnetic field is assumed to scale with radius as $B_{\phi} \propto 1 / r$, although this is not essential (we have run models for which $B_{\phi}=0$ and still observe outflows). The initial radial and vertical magnetic field components in the disk are continuous with the field in the corona. There is no disk, rotation, or toroidal field inside the radius $r_{i}$.

All length scales and speeds in the simulations are measured in units of innermost disk radius $r_{i}$ and the Kepler speed at that radius, $v_{K, i}$, so that results can be scaled to a central object of any desired mass. All time scales are measured in units of $t_{i}=r_{i} / v_{K, i}$. Typical conditions in protostellar or AGN disks are, for the former, $r_{i} \simeq 3$ stellar radii $=0.04$ astronomical units (AU), and for the latter, 10 black hole radii.

Our numerical simulations are governed by five parameters defined at $r_{i}$. The ratio of the thermal gas pressure to the magnetic field pressure, $\equiv \beta_{i}$; the square of the ratio of the Kepler speed to the sound speed, $\equiv \delta_{i}$; the ratio of the disk density to the coronal density, $\equiv \eta_{i}$; and the disk's toroidal to poloidal field ratio, $\equiv \mu_{i}$. For all Figures, the injection speed is $v_{i n j ., i} / v_{K, i}=10^{-3}$; and $\left(\beta_{i}, \delta_{i}, \eta_{i}, \mu_{i}\right)=(1.0,100.0,100.0,1.0)$. All 
the models are run at high spatial resolution of $(500 \times 200)$ zones. We use inflow boundary conditions at the disk surface and open outflow conditions (i.e., zero normal gradient) on the remaining boundaries (except along the axis of symmetry, coinciding with the disk axis, taken to be reflecting (i.e., zero normal velocity)).

\section{Stationary outflows}

We show a series of snapshots of the flow in Fig. 1 that illustrate the eventual realization of a stationary hydromagnetic outflow. As expected, the first stage of evolution in our simulations is a brief transient as a torsional Alfvén wave front propagates through the corona from the disk surface. Thereafter the flow either relaxes to a steady state outflow pattern, or an episodic outflow is established. In Fig. 1a, we plot surfaces of constant magnetic flux for an assumed potential field configuration and density isocontours at the initial instant. The evolution of the two-dimensional magnetic field structure is shown in Figs. $1 \mathrm{~b}$ and 1c, at 100 inner time units, and at 400 time units by which time the working surface of the jet has long left the end of our mesh. At time 100, the region behind the working surface of the flow has already achieved a cylindrically collimated state while the region ahead of the working surface still retains the original field structure. At time 400, the entire flow in our mesh has been collimated. Cylindrical collimation is predicted for steady MHD winds (Heyvaerts and Norman 1989). In Fig. 1c; we plot the location of Alfvén surface (filled hexagons), as well as the FM surface (stars). There is good agreement between our numerical results and steady state theory for the position of the Alfvén surface as is shown in Fig. 1d. At the base of the outflow, the flux surfaces in Fig. 1c (or equivalently, the stream-lines) make an angle of $\simeq 55^{\circ}$ with respect to the disk across most of its surface. This geometry satisfies the outflow condition of steady state theory that $\theta_{o} \leq 60^{\circ}$.

The two-dimensional distribution of the toroidal magnetic field and vertical outflow speed as compared with the density is shown in OPS and OPI. An obvious bow shock traces the working surface of the outflow with respect to the undisturbed corona. There is also a highly collimated jet-like stream of gas that starts at the disk surface. The comparison of the jet density and toroidal magnetic field shows that the outflow density is delimited by the region of strong toroidal field. The physical reason for this is the strong radial pinch force that is exerted towards the jet axis by the magnetic force arising through the combination of a dominant toroidal field, and its associated current which flows primarily along the outflow axis. The highest speed gas is nearest the outflow axis with lower speeds at larger radii (see Fig. 2, OPS). 
a
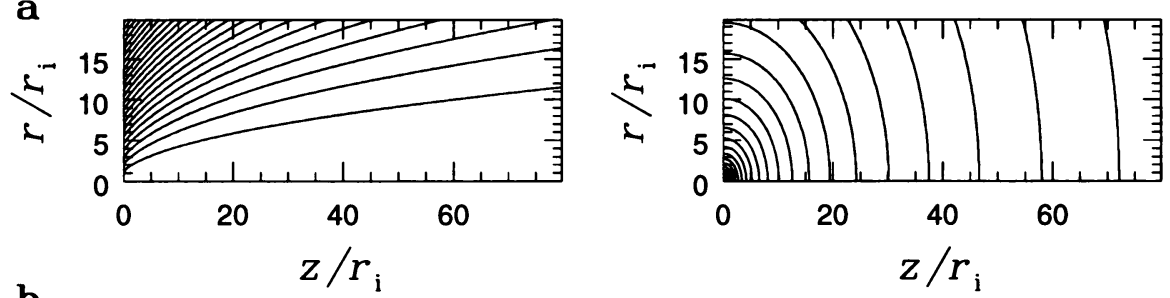

b
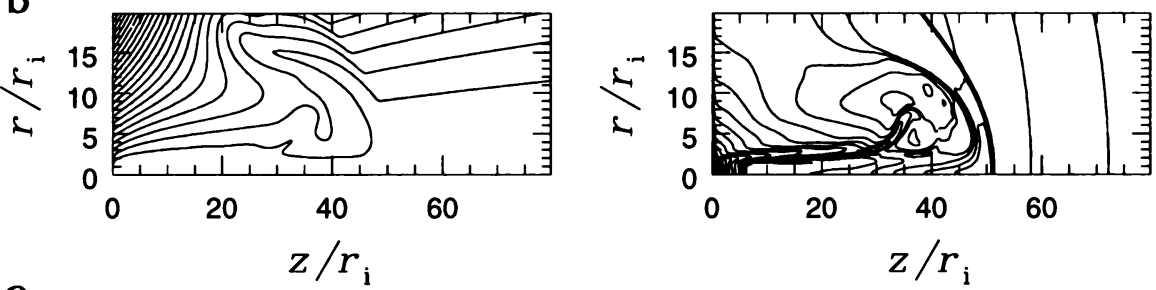

C
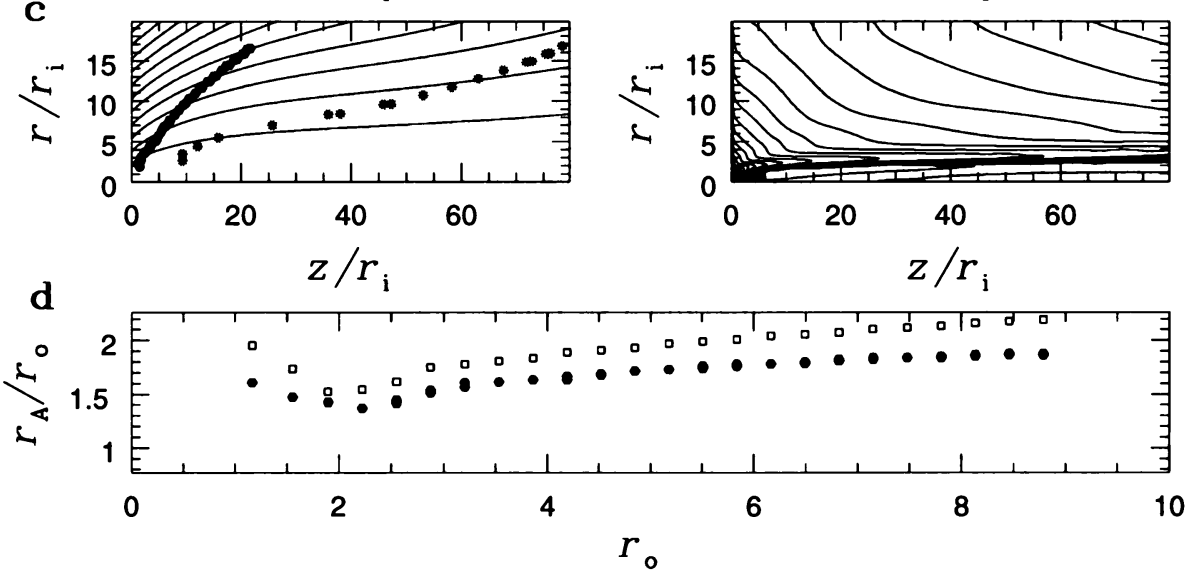

Figure 1. In all figures, the axis of symmetry is plotted horizontally and the disk surface, vertically. Frame 1a. (top) The initial magnetic configuration, shown in the left panel, is the potential field. The right panel shows the initial isodensity contours of the corona. Frames b and c; evolution of the initial magnetic and density structure (the left and right panels respectively) at 100 and 400 inner time units. Frame c; location of the Alfven critical surface (filled hexagons) and of the FM surface (stars). Frame d; comparison of the Alfvén lever arm $r_{A} / r_{o}$ for each field line in the simulation (filled hexagons), with predictions of steady state theory (squares). (Adapted from OPS).

In order to show the details of the acceleration mechanism, we plot in Fig. 2 the values of various physical quantities at increasing distance along a fiducial field line, anchored near $r_{o}=r_{i}$. Fig. 2a shows that the Alfvén and FM Mach numbers achieve values of 5 and 1.5 respectively. The ratio of the toroidal to poloidal field strength builds from an initial value of unity to 2 (Fig. 2b). Thus, beyond the FM surface, the toroidal magnetic field 

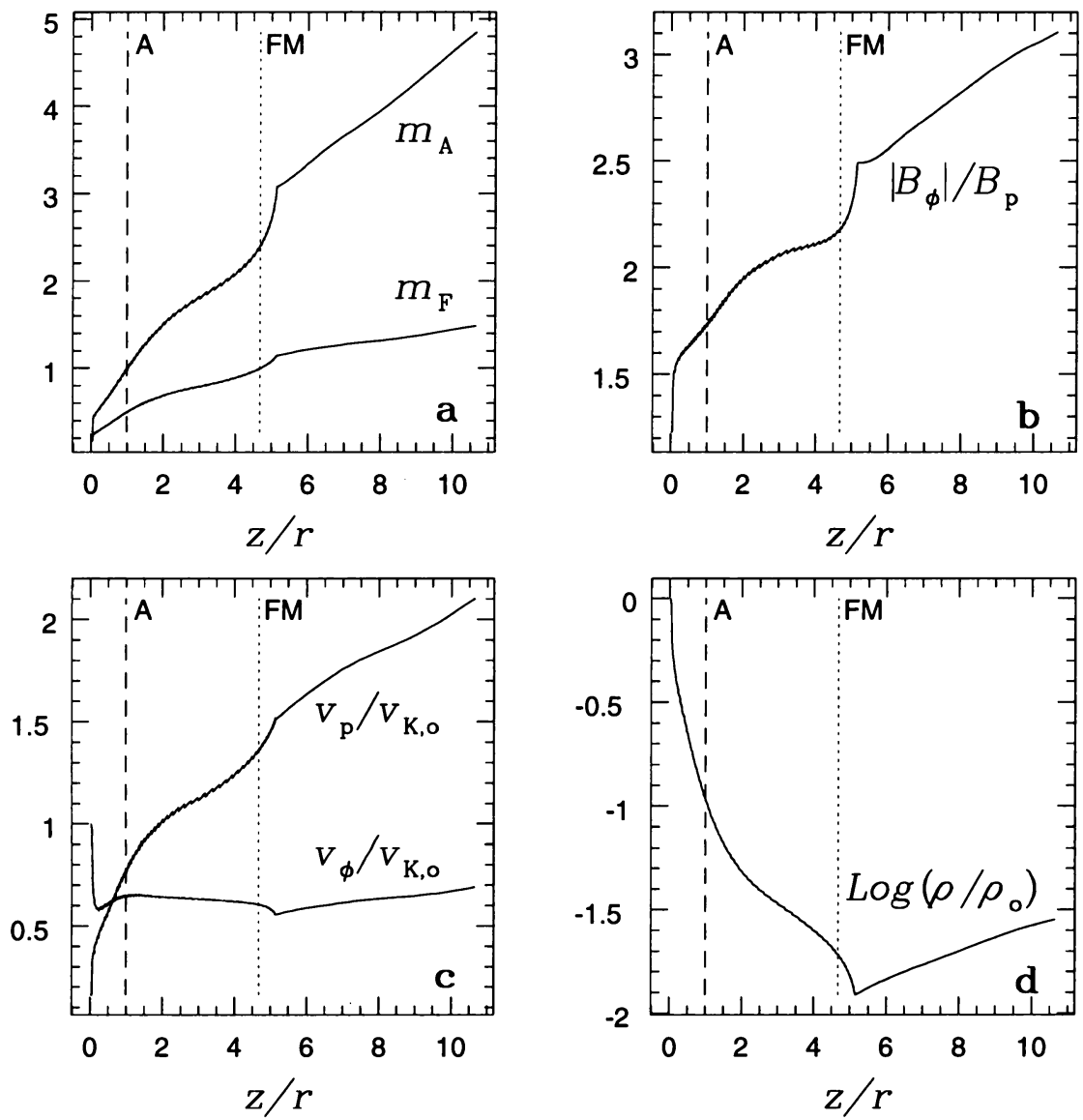

Figure 2. Physical quantities along field line anchored at $r_{i}$ plotted against $z / r=\operatorname{cotan}^{-1}(\psi)$ where $\psi$ is the polar angle as measured from the origin of the coordinate system. Frame 2a; Alfvén Mach numbers $m_{A}$ and the FM numbers $m_{F}$, Frame $2 \mathrm{~b}$, ratio of the toroidal to poloidal magnetic field along field line; Frame 2c, poloidal velocity $v_{p}$ and toroidal velocity $v_{\phi}$, in units of the Kepler speed at the footpoint of the field line; and Frame 2d, the density $\rho$ in units of the density at the footpoint of the field line. The position of the Alfven (marked A) and FM points (marked FM) on the field line are also given. (Adapted from OPS)

dominates the dynamics of the jet. Fig. 2c shows that the poloidal speed of the gas along a field line starts at its input value of a thousandth of the Kepler speed at the base of that field line $\left(v_{K, o}\right)$, reaches 1.5 times this value at the FM point, and continues to accelerate to $2.1 v_{K, o}$ at the end of the grid. Thus, flow speed from our fiducial, low mass young stellar object 
reaches upwards of $220 \mathrm{~km} \mathrm{~s}^{-1}$, comparable to observed values. The density of the flow decreases as one moves outwards along the field line because of the divergence of the flow streamlines (Fig. 1c).

The outflow achieved in this simulation has properties predicted by steady MHD disk-wind theory. The acceleration of material occurs in two separate stages. In stage 1 (the region along a field line between the disk and the FM point) the centrifugal effect dominates. In stage 2, (region beyond the FM point), the flow speed scales as $v_{z} \propto z$, which is theoretically expected when outflow becomes super-Alfvénic (supersonic for hydrodynamic jet models; see Raga and Kofman 1992).

This simulation produces mass, momentum and energy flux rates across the outer axial boundary of our simulation, comparable to those for AGN and protostellar outflows (see OPI). The disk accretion rate, deduced from our mass outflow rate using steady state theory, is $\dot{M}_{a} \simeq 6 \dot{M}_{w}$.

\section{Episodic outflow}

The field lines in this simulation comprise an initially uniform and vertical magnetic field structure that penetrates the disk and overlying corona. According to steady-state models, this configuration is unfavourable for launching an outflow. In the four snapshots taken at 100, 200,300, and 400 time units, Fig. 3 shows that outflow is nevertheless launched from the accretion disk for reasons we describe below. The density structure is shown in the left panels, and the toroidal field contours on the right. The highly collimated, jet-like flow has a density structure that is dominated by discrete knots. At time 100 and 200, the working surface of the outflow is clearly evident. The working surface in this simulation has the shape of a cone that advances into the flow with a swept-back bow shock along its flanks. Such nose cone structures have been seen in pure MHD jet simulations (Clarke et al. 1986, Lind et al. 1989). This surface leaves our grid long before 400 time units have elapsed.

The density structure in this simulation never achieves steady state. We have run simulations up to 1000 time units and find that the episodic nature of the flow persists unabated. The density contours show that the jet is strongly confined near the axis. We find that the associated toroidal field in the flow is tightly anti-correlated with the knots; high (low) density regions are associated with the lowest (highest) toroidal field strength. The average speed of material in this outflow is $0.5 v_{K, i}$, with the highest outflow velocities $\simeq v_{K, i}$. Although this velocity is not as large as is sometimes observed on larger scales in real outflows, our simulations explore only a limited region of physical and parameter space. Movies of this simulation (http://www.physics.mcmaster.ca/Grad_Ouyed/ROuyed.html) show that 

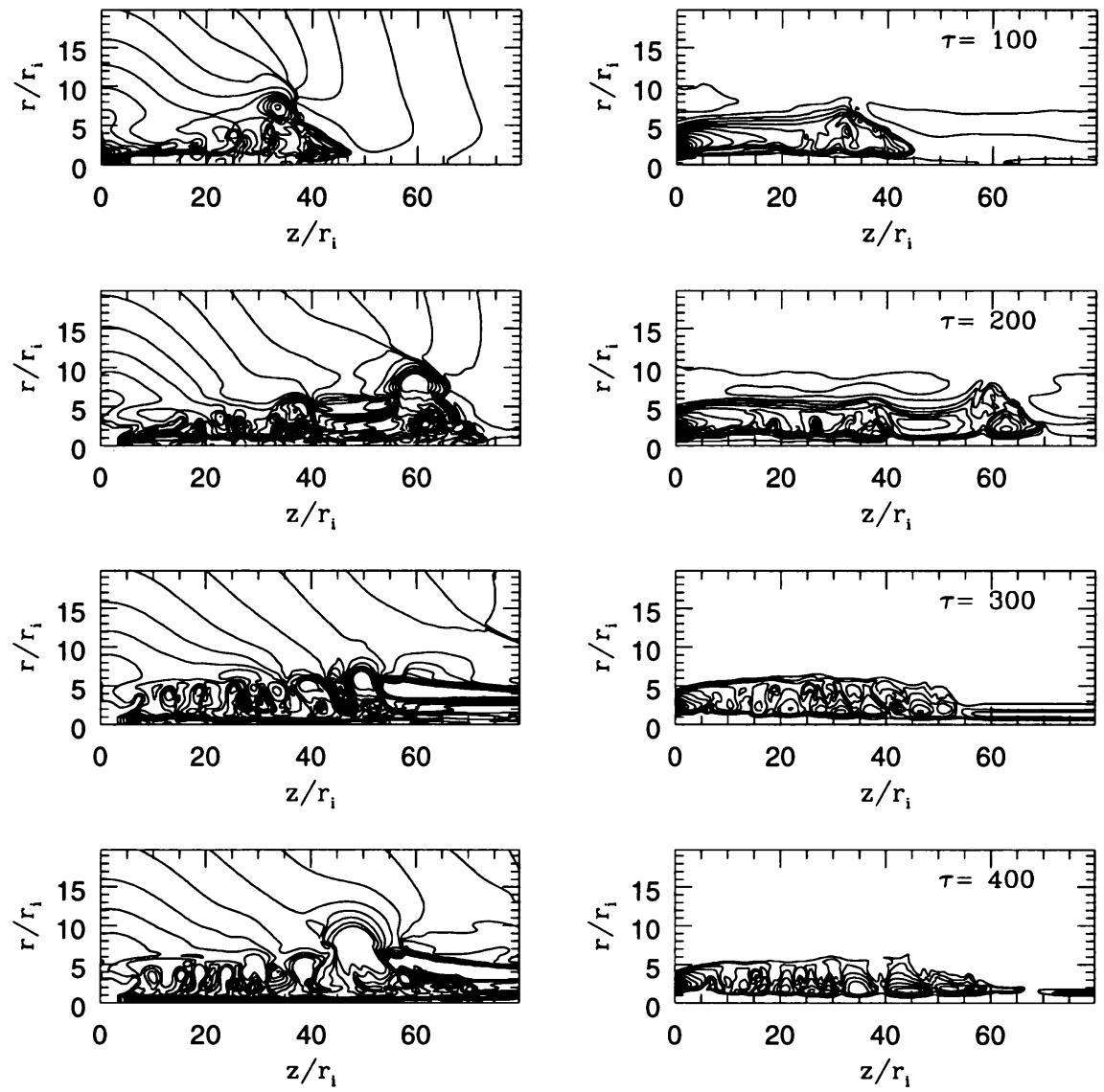

Figure 3. Jet density (left) and toroidal field (right) showing evolution of episodic outflow at 4 different times. 20 logarithmically spaced contour lines are shown for density, 20 equally spaced contours for $B_{\phi}$. (Adapted from OPII).

the knot generator stays fixed in space and that the knots, once formed, persist as coherent structures that propagate down the length of the jet.

To explore the knot generating region further, we performed a simulation at 4 times the axial and twice the radial resolution, but otherwise identical to that shown in Fig. $3 ;(10 \times 20) r_{i}$.

In Figure 4, we show the magnetic field configuration of the outflow at times 37.2, 42.6, and 48.0 during which the new knot (B) is formed (knot A moves away from the generating region as knot $\mathrm{B}$ is formed). This process takes a time $\tau_{k n o t} \simeq 11$ time units. The knots are produced at a distance 
of $z_{k n o t} \leq 6-7 r_{i}$ from the central source. Since the variation in axial velocity associated with the knots is supersonic, they are associated with MHD shocks (see OPII) . Comparison of frames a and $\mathrm{c}$ shows that knots are spatially separated by a distance of $\delta z_{k n o t} \simeq 5 r_{i}$ and thus move at a speed of $\simeq 0.5 v_{K, i}$ out of this generating region.

The plots in the right hand panels of Fig. 4 reveal how the outflow and its knots are generated. A narrow radial region of field lines in the innermost parts of the disk has been opened up, making an angle of $50^{\circ}$ with respect to the disk surface. This narrow region drives the outflow and is produced by the toroidal field which is strongest in the inner regions of the outflow where the underlying Kepler rotation is the largest. A radial, outwardly directed, toroidal magnetic pressure gradient is produced by the newly created toroidal field and this pushes open the field lines setting up a condition favourable for outflow. The knots are produced in a region of the flow beyond the Alfvén (marked on the field lines in Fig. 4) and FM surface in the outflow.

The size of the wind production region on the surface of the disk depends upon the ease with which field lines can be pushed aside by the toroidal field pressure (Ouyed 1996). The rigidity of the field is measured by the $\beta$ parameter and is very different for our two cases. For the more magnetically dominated episodic case, the magnetic pressure associated with the self-generated toroidal field in the narrow band of outflowing gas has a local maximum so that the pressure force, acts both radially outwards, and inwards towards the axis. The accelerating outflow, on encountering the inwardly directed pressure gradient, is reflected back towards the axis. Because the outflow is rotating however, the gas spins up as it moves inwards and reflects off an inner "centrifugal barrier" when it reaches a radius comparable to its footpoint radius (see Fig. 4 in OPII). The resulting nearly harmonic oscillation in the width of the flow (Sauty and Tsinganos 1994) between these two "barriers" must, by mass conservation, lead to variations in flow velocity (much like a constricted garden hose). These variations in flow speed rapidly steepen into fast MHD shocks (Gomez de Castro and Pudritz 1993, Ouyed and Pudritz 1993, Ouyed and Pudritz 1994).

Estimating that the toroidal Alfvén speed in our simulation is $v_{A, \phi} \simeq$ $0.5-0.6 v_{K, i}$, and the width of the jet to be $\delta r_{j} \simeq 3-4 r_{i}$, the oscillation period of the jet is $t_{o s c}=2 \delta r_{j} / v_{A, \phi} \simeq 11-13 t_{i}$, which is indeed the knot production time scale. By contrast, the initial magnetic pressure in the steady flow case drops significantly as one moves outwards from the axis. The accelerating outflow does not encounter a strongly magnetically overpressured outer barrier, and continues to expand radially finally achieving a quiet, cylindrically collimated state. 

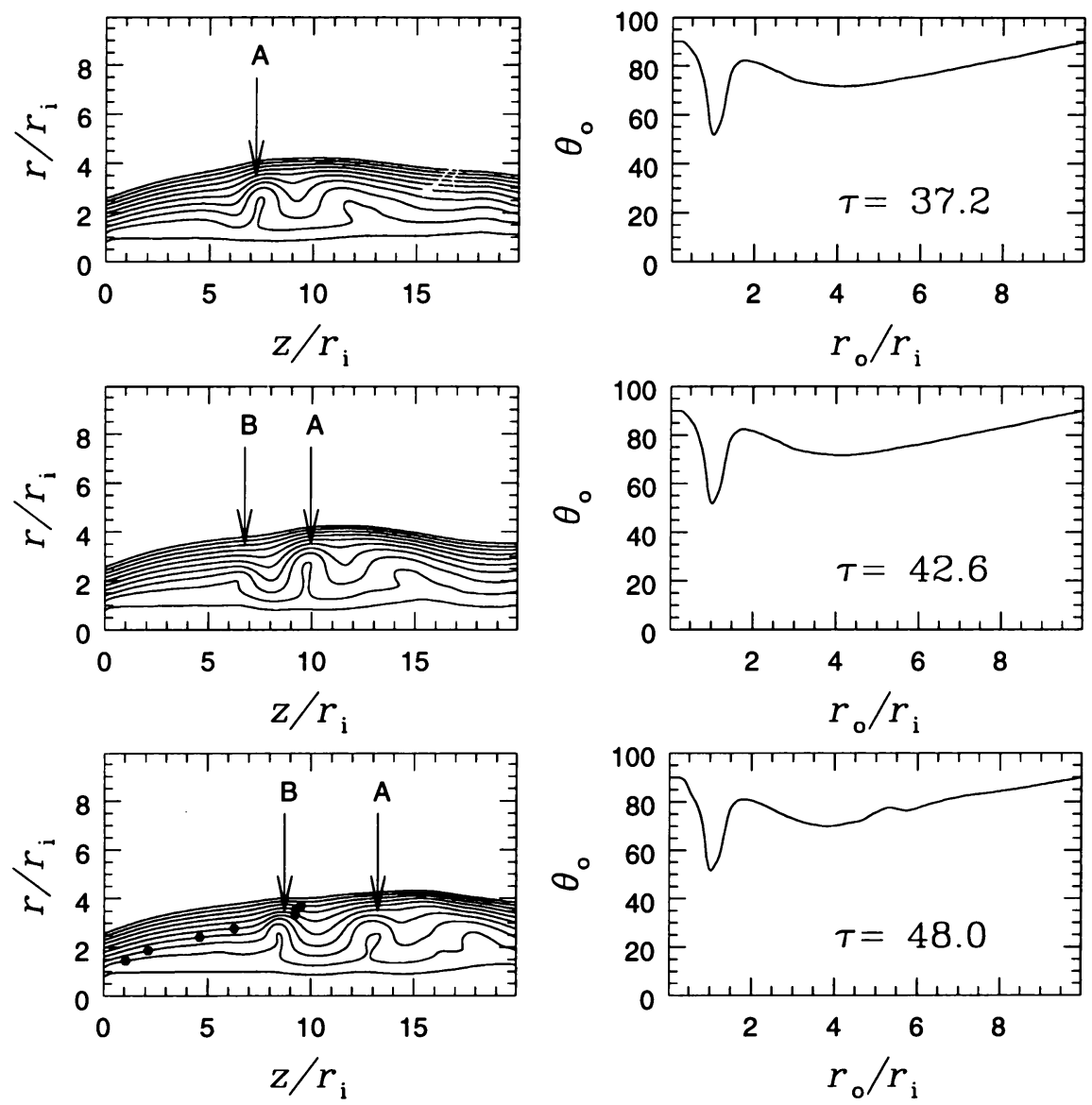

Figure 4. The left panels show the magnetic field structure of the knot generating region, at the three times; $37.2,42.6$, and 48.0 inner time units. The right panels show the angle $\theta_{o}$ of field lines at the base of the flow, with the disk surface, at these times. Note the narrow band of field lines which is sufficiently opened $\left(\theta_{o} \leq 60^{\circ}\right)$ so as to drive the outflow. Only field lines involved in the knot generation process are shown; field lines at larger disk radius stay reasonably vertical as seen in the right panels. (Adapted from OPS.)

\section{Transition from Episodic to Stationary Flow}

In Ouyed (1996) and OPIII, we have investigated the importance of toroidal field in producing episodic jet behaviour. We found that when the number $N=B_{\phi, i}^{2} / 8 \pi \rho_{i} v_{i n j .}^{2}>1(N$ is the ratio of the confining toroidal field pressure to the ram pressure of gas injected into the corona; this ratio is a 
constant for the uniform field configuration), then the outer toroidal barrier is strong and the flow is episodic. However, if $N<1$, then the toroidal magnetic pressure is overwhelmed and a stationary flow should result. We performed a series of eight simulations identical to those shown in Figs 3 and 4, except that the injection speed in the initially uniform vertical field configuration was varied from a value of 0 , to a maximum of $10^{-2} v_{K, i}$. The results of these calculations are shown in the accompanying Table (adapted from OPIII). The transition between episodic and stationary flow appears to respect this criterion very well.

TABLE 1. Effect of Varying the Injection Velocity $v_{i n j}^{a}$ (adapted from OPIII)

\begin{tabular}{lllllllll}
\hline$\frac{v_{i n j} / v_{K}}{10^{-3}}$ & $\mathrm{~W}^{b}$ & $N_{K}^{c}$ & $\frac{z_{g e n .}}{r_{i}}$ & $\frac{\delta r_{w i n d}}{r_{i}}$ & $\frac{\Delta z_{k n o t}}{r_{i}}$ & $\frac{\Delta t_{k n \text { ot }}}{t_{i}}$ & $\frac{\bar{v}_{\infty}}{v_{K, i}}$ & $\left(\frac{B_{\phi}^{2} / 8 \pi}{\rho v^{2}}\right)^{d}$ \\
\hline 0.0 & 3 & 1 & 4 & - & - & - & 0.3 & $\infty$ \\
0.1 & 2 & 2 & 5 & 1 & 7 & 15 & 0.3 & $3.6 \times 10^{3}$ \\
0.5 & 2 & 3 & $7-8$ & $3-4$ & $5-7$ & $11-13$ & 0.5 & $1.45 \times 10^{2}$ \\
1.0 & 2 & 3 & $7-8$ & $3-4$ & $5-7$ & $11-13$ & 0.5 & $3.6 \times 10^{1}$ \\
5.0 & 1 & 0 & - & 10 & - & - & 0.8 & 1.45 \\
10 & 1 & 0 & - & 10 & - & - & 0.9 & 0.36 \\
\hline
\end{tabular}

${ }^{a}$ The rest of the parameters are the standard ones. Namely, $\beta_{i}=1.0, \delta_{i}=100.0, \eta_{i}=$ 100.0 and $\mu_{i}=1.0$.

${ }^{b}$ Wind properties: $1 \equiv$ steady; $2 \equiv$ episodic; $3 \equiv$ transient.

${ }^{c}$ Number of Knots: Total time of simulation $t_{S}=50 t_{i}$. The simulated region is $(r \times z)=$ $(10 \times 20) r_{i}$ with a resolution of $(200 \times 500)$ cells.

${ }^{d}$ Estimated at the disk surface (injection values).

\section{Conclusions}

The existing numerical simulations, including our own, only explore a small part of parameter space. To date, the published simulations assume ideal MHD and some polytropic equation of state. Our own work simulates a region close to the central engine which is still a decade and a half smaller than could be resolved by HST. The two assumptions of our simulations which are most important however, are that of axisymmetry as well as the fixed nature of the disk boundary conditions.

The main effect of reducing the dimensionality of the dynamics is to eliminate some modes of MHD instabilities, such as the potentially important non-axisymmetric modes. These modes can play an important role in the dynamics of the winds. The major constraint is that the accretion disk is not allowed to respond to the changing torques exerted by the jet. 
This assumption is reasonable as long as disk evolution is slower than the evolution of the jet. Given that the Alfvén crossing time in the jet is much shorter than in the disk, this is a plausible assumption. Thus, in spite of their limitations, our simulations may provide a good first step towards a detailed theory of astrophysical jets as they are observed.

Acknowledgements: The authors are grateful to the organizers for the opportunity to present this work at this most stimulating conference. We are indebted to Jim Stone, David Clarke, and Patricia Monger for stimulating conversations and assistance. REP thanks CITA for the stimulating environment and support that he enjoyed during a sabbatical leave there as this article was being written. The research of REP is supported by an operating grant from the Natural Sciences and Engineering Research Council of Canada.

\section{References}

André, P., Ward-Thompson, D., \& Barsony, M. 1993, Ap. J., 406, 122

Balbus, S. A. \& Hawley, J. F. 1991, Ap. J., 376, 214

Beckwith, S. V. W., Sargent, A. I., Chini, R. S., \& Güsten, R. 1990, A. J., 99, 924

Bell, A. R. \& Lucek, S. G. 1995, MNRAS, 277, 1327

Blandford, R. D. 1990, In SAAS Fée Lectures on Advanced Astrophysics, Active Galactic Nuclei, eds. R. D. Blandford, H. Netzer and L. Woltjer, Berlin:Springer

Blandford, R. D \& Payne, D. R 1982, MNRAS, 199, 883 (BP)

Cabrit, S., \& André, P. 1991, Ap. J., 379, L25

Calvet. N., Hartmann, L., \& Kenyon, S. J. 1993, Ap. J., 402, 623

Cao, X. \& Spruit, H. C. 1994, A\&A, 287, 80

Clarke, D. A., Norman, M. L. \& Burns J. O. 1986, Ap. J., 311, L63

Collin-Souffrin, S. 1992, In AIP Conf. No. 254, Testing the AGN Paradigm, eds. S. S. Holt, S. G. Neff and C. M. Urry, p. 119

Curry, C., Pudritz, R. E., \& Sutherland, P. G. 1994, Ap. J., 434, 206

Edwards, S., Ray, T. P. \& Mundt R. 1993, In Protostars and Planets III, eds. E. Levy and J. Lunine, University of Arizona Press, p. 567

Eislöffel, J. \& Mundt, R. 1994, A\&A, 284, 530

Ferreira, J. \& Pelletier, G. 1995, A\&A, 295, 807

Gomez de Castro, A. I., \& Pudritz, R. E. 1993, Ap. J., 409, 748

Heyvaerts, J. \& Norman, C. 1989, Ap. J., 347, 1055

Hjellming, R. M, \& Rupen, M.P. 1995, Nature, 375, 464

Königl, A. 1989, Ap. J., 342, 208

Königl, A., \& Ruden, S. P. 1993, in Protostars and Planets III, E. H. Levy and J. I. Lunine eds. (Tucson: University of Arizona Press), 641.

Li, Z.-Y. 1995, Ap. J., 444, 848

Lind, K. R., Payne, D. G., Meier, D. L. \& Blandford, R. D. 1989, Ap. J., 344, 89

Lovelace, R. V. E., Wang, J. C. L., and Sulkanen, M. E. 1987, Ap. J., 315, 504

Lubow, S. H., Papaloizou, J. C. B., \& Pringle, J. E. 1994, MNRAS, 268, 1010

Masson, C. R. \& Chernin, L. M. 1992, Ap. J., 387, L47

Ouyed, R. 1996, Ph.D. Thesis, McMaster University

Ouyed, R., \& Pudritz, R. E. 1993, Ap. J., 419, 255

Ouyed, R., \& Pudritz, R. E. 1994, Ap. J., 423, 753

Ouyed, R., \& Pudritz, R. E. 1997a, Ap. J., June 20 issue (OPI)

Ouyed, R., \& Pudritz, R. E. 1997b, Ap. J., August 1 issue (OPII) 
Ouyed, R., \& Pudritz, R. E. 1997c, MNRAS, submitted (OPIII)

Ouyed, R., Pudritz, R. E., \& Stone, J. 1997, Nature, 385, 409 (OPS)

Parker, N. D., Padman, R., \& Scott, P. F. 1991, MNRAS, 252, 442

Pelletier, G. \& Pudritz, R. E. 1992, Ap. J., 394, 117

Pudritz, R. E. 1981, MNRAS, 195, 881

Pudritz, R. E., McLaughlin, D. E., \& Ouyed, R. 1997, in A.S.P. Conference Series: 12th Kingston Conference on Theoretical Astrophyiscs, Computational Astrophysics, D.A. Clarke \& M.J. West eds. (San Francisco: ASP), in press.

Pudritz, R. E., Pelletier, R., \& Gomez de Castro, A. I. 1991, in Physics of Star Formation and Early Stellar Evolution, C. Lada and N. Kylafis eds. (Dordrecht: Kluwer), 539.

Pudritz, R. E., Wilson, C. D., Carlstrom, J. E., Lay, O. P., Hills, R. E., \& WardThompson, D. 1996, Ap. J., 470, L123

Raga, A. C. \& Kofman, L. 1992, Ap. J., 386, 222

Reipurth, B. 1989, In Low Mass Star Formation and Pre-Main-Sequence Objects, ed. Bo Reipurth, Garching: ESO, 247

Reys-Ruis, M. \& Stepinski, T. 1996, Ap. J., 459, 653

Sauty, C., \& Tsinganos, K. 1994, A\&A, 287, 893

Shibata, K., \& Uchida, Y. 1986, PASJ, 38, 631

Shu, F. H. 1977, Ap. J., 214, 488

Shu, F. H., Najita, J., Wilkin, F., Ruden, S.P., \& Lizano, S. 1994, Ap. J., 429, 781

Snell, R. L., Loren, R. B., \& Plambeck, R.L. 1980, Ap. J. L., 239, L17

Stapelfeldt, K. R. 1996, private communications.

Stone, J. M., \& Norman, M. L. 1992a, Ap. J. S., 80, 753

Stone, J. M., \& Norman, M. L. 1992b, Ap. J. S., 80, 791

Stone, J. M., \& Norman, M. L. 1994, Ap. J., 420, 237

Stone, J. M., Hawley, J. F., Gammie, C. F., \& Balbus, S. A. 1996, Ap. J., 463, 656

Tout, C. A., \& Pringle, J. E. 1992, MNRAS, 259, 604

Uchida, Y., \& Shibata, K. 1985, PASJ, 37, 515

Ustyugova, G. V., Koldoba, A. V., Romanova, M. M., Chechetkin, V. M. \& Lovelace, R. V. E. 1995, Ap. J. L., 439, L39

Wardle, M., \& Königl, A. 1993, Ap. J., 410, 218 